\title{
COOPERATIVE STRATEGIES FOR CHILDREN WITH AUTISM SPECTRUM DISORDERS IN INCLUSIVE ROBOTICS ACTIVITIES
}

\author{
Evaggelia Tsiomi \\ Include, Greece \\ Andromachi Nanou \\ Include, Greece
}

\begin{abstract}
A qualitative, action research study has been conducted to investigate cooperative learning strategies for children with ASD and typical development during educational robotic activities. The participants were 4 children, 2 with ASD and 2 with typical development, around the ages of 12-14. The sessions were held at "School for all: Tokei Maru" in Thessaloniki, once a week, for 3 months. Observation protocols, researcher's diary, and interviews were used for data collection. Children with ASD, although they had a strong motivation to participate in robotic activities, exhibited disruptive behaviors. The cooperative learning strategy was not effective. Self-regulation strategies have proven helpful in limiting the disrupting behaviors of children with ASD and in supporting cooperative learning.
\end{abstract}

Keywords: inclusion, lego type robots, ASD children, cooperative strategies self regulated strategies.

\section{Introduction}

Children with Autism Spectrum Disorder (ASD) encounter obstacles in communication and socialization. Their opportunities to be engaged in social interactions and friendliness relationships are limited due to their difficulties in understanding other's thoughts, desires, and feelings, in initiating the communication and in applying effective social and communication strategies (Rogers, 2000; Mc Connell, 2002; Owens, Gordon, \& Baron-Cohen, 2008).

Most of the intervention programs, which were addressed to children with ASD aim at improving social skills and social interaction (Walton \& Ingersoll, 2013). In recent decades, there is a significant interest in investigating how the educational or social robotic programs would support the development of social skills and interactions of autistic children. Particular interest has been developed in investigating how children with ASD would be supported by cooperation during their participation in inclusive educational robotic activities. Hinchliffe and his colleagues (2016) in Australia, investigated the effects of the participation of children with ASD in inclusive, after school robotics clubs. According to their 
findings, 7-10 years old children with ASD demonstrated an increased awareness of peer social networks, experienced greater benefit in terms of their integration and acceptance into the social network of the club, compared to their same-aged peers (Hinchliffe, Saggers, Chalmers, \& Hobbs, 2016). Effective collaboration strategies for inclusive robotic activities are being investigated, in recent decades, as robots have also a great educational value. A number of methods, based on the framework of social constructivism, such as cognitive mentorship and scaffolding, provide opportunities to develop communication, social, metacognitive, problem-solving or critical thinking skills in cooperative learning environments (Ching - Ching, Pei-Li, \& Kuo-Hung, 2013). In robotic activities all students work together in all aspects, designing, assembling, programming, testing, debugging, and modifying. Children, working together, need to use social skills such as integrating others' ideas, negotiating and coordinating viewpoints, and seeking agreement. Effective collaboration is under scripted but switchable roles (Denis \& Hubert, 2001; Wang \& Zhong, 2018).

Cooperative learning (CL) has been shown to increase levels of social engagement in inclusive school environments. Peers involved in CL interventions with students with disabilities have displayed significant increases in social acceptance to peers not involved in CL groups (Grey, Bruton, Honan, McGuiness, \& Daly, 2007). Specific cooperation learning (CL) strategies help children with ASD to interact in a team and therefore to gain opportunities to improve their social skills (Leman, 2015; Reynolds, Bendixen, Lawrence, \& Lane, 2011). Research concerning cooperative strategies for children with ASD in inclusive robotic activates has to be investigated as the benefits have been documented from Australia (Hinchliffe et al., 2016). Self-regulation strategies help children with ASD to regulate their behaviors. Are processes that would be activated by students to focus and sustain cognitions and behaviors, which are systematically oriented toward the attainment of their goals (Reid, Mason, \& Asaro-Saddler, 2013). Action research has brought out the need of self-regulated strategies to help children with ASD to organize their behaviors in robotic activities (Nanou, Chenine, \& Oikonomou, 2019). The aim of this study was to investigate the effects of cooperative learning strategies in combination with Selfregulated strategies in children with ASD as they cooperate with typical peers in inclusive robotic activities.

\section{Research Methodology}

Action research has been chosen as the most appropriate research methodology for investigating effective cooperative learning strategies in an authentic learning environment. Action Research, through creative interaction and authentic experience, leads to theoretical and practical knowledge (Magos, \& 
Panagopoulou, 2008; McNiff, 1999). The action research is executed through a series of research circles. The end of the first research circle is the beginning of the second. Every circle involves specific stages:
a) plan,
b) action,
c) observe,
d) reflect.

Place and time of research. The action research lasted 3 months (16/1/2017 to 16/3/2017) with frequency once a week. The day, time, and duration were always the same (60'). The place was the "School for all: Tokei Maru” a non-formal, after school learning inclusive environment, in Thessaloniki, Greece

Participants. 4 children, 2 with ASD at level 1 according to DSM 5, Giannis and Fotis, and 2 children of typical development, 12-14 years old, participated in robotic activities (DSM 5, 2013). As revealed by the parents' interviews, prior to the first meeting of the robotics group, the ASD children exhibited the following characteristics: Giannis expressed himself syntactically well, but his speech was monotonous quirkiness. His intonation often was the cause of negative comments by his peers. He faced significant difficulties focusing on one particular activity, in interpersonal relationships and social intercourse and therefore he seems to have no friends. Fotis, has advanced cognitive skills, limited and selective oral speech and difficulty in visual contact. He found difficulties to socialize and preferred individual play. He didn't work with peers, didn't ask for help, or offer help to peers and had difficulty in adhering to instructions and rules. Robotics was the favorite hobby of both Giannis and Fotis.

The typical development participants were selected by a list of children, who were interested to join robotics activities in School for all "Tokei Maru". The selection criterion was their previews experience in collaboration with children with ASD at level 1 . The two children that they have chosen to participate had the previous years in "School for all: Tokei Maru" cooperate well with children with ASD.3 special pedagogues, volunteers, were participated in an action research study. One of them has been specialized in coding and robotics and was the coordinator. The other two were external observers

\section{Teaching methodology -Strategies}

The researchers planned to divide the participants in 2 groups - pairs, so as each pair to include one child with ASD and one typical peer. The pairs were selected by the coordinator. In each session they had to collaborate under scripted and switchable roles to assemble 5 steps, as imprinted in the manual building instructions. Think Share Pair cooperative strategy planned to be used in robotic activities. The challenge for each pair was to discuss and take decisions about 
specific roles and the way they switched them during their assembling cooperation. The coordinator, the special pedagogue, planed to challenge children to think of their role and then to share with their pair. Then the pairs had to discuss their decisions and reform their decisions in the light of the other pair ideas (Lyman \& Frank, 1987). So a specific self-instruction strategy to support the usage of CL planned to be used. Self-instruction strategies have been proved helpful (Wehmeyer et al., 2003) Social stories were used to teach children the component of SMS strategy. The components of the strategy were presented in a power point presentation with pictures ready to read from all children with the aim to present to prepare more precise the children with autism to use them during activities. Social story approach is extremely effective for social skill education of children with ASD (Gray, 1998). Additionally the components presented and posted to make it accessible to children in the "School for all: Tokei Maru" so that the SMS strategy can be seen by all children when needed. The strategy had three components: $\mathrm{S}=$ staring in my pair's position, $\mathrm{M}=$ Manage assembling with my pair, $\mathrm{S}=$ Share to the others. The components of SMS strategy and the pictures they have been used are presented bellow (Figure 1).

\begin{tabular}{|c|c|c|}
\hline \multicolumn{3}{|c|}{ SMS Strategy Components } \\
\hline S & M & S \\
\hline Staring at my pair's opinion & Manage with my pair & \\
\hline
\end{tabular}

Figure 1 SMS self-instruction strategy to support cooperative learning (CL)

\section{Data Collection}

For data collection have been used:

1) Parents' semi-structured interviews, before action research, in order for the researchers to be informed about the child's psychological characteristics,

2) research diary which was completed after each session by the coordinator researcher,

3) Strategy Deviation Assessment Protocol (SDAP). SDAP assess the use of strategy components. It is a descriptive assessment scale which was developed for the purpose of the study. It is based on assessment Protocols that was used to asses social skills during school lessons by children with Learning disabilities (Drosinou, 2009). The assessment 
criteria of SDAP were the 3 components of the strategy SMS. Strategy components use were assessed with 0 point $=$ use and $-1=$ no use. The main point of 3 components strategy use of SMS strategy in 5 steps was given the main deviation of strategy use in every session. The usage of SMS strategy components by the ASD children was assessed by the 2 external observers in every session. The results of strategy use at every session, discussed for feedback between the researchers. The participant and external observers evaluate the process and redefine any changes. The changes that have to be done define the circles of the action research.

\section{Findings Process of the action research}

1st circle: Action at the $1^{\text {st }}$ session, Giannis and Fotis faced difficulties to be engaged in discussions concerning their role in assembling. Fotis was very impatient. He didn't even look at his partner while he was trying to assemble. According to the coordinator researcher's diary, both Fotis and Giannis exhibited non-creative forms of interaction such as negative verbal comments and gestures. "The typical children bothered by disrupted behaviors and after 2 steps in assembling the pedagogists decided to interrupt the assembling to discuses and present the SMS Strategy. A social story of SMS components, through power point, presented in the interactive table. After the presentation children continue to assemble the next 3 steps. Every session proceeded with The mean of the SMS use was $-4,0$ at the first 2 sessions for both children. Gradually children improve their focus and cooperation but they really didn't ask opinions and they use to manage the robot themselves. It is important that typical children found more difficult sometimes, as the coordinator write, to find the parts and assemble the robots than children with ASD.

Observe: According to the participant-researcher's diary and the SDAP protocols, children with ASD at the first 5 sessions presented deviation of strategy use with a gradually improvement from $-4,0$ to $-3,5$ points. Fotis and Giannis, in both groups although they were both improve their cooperation skills during the 5 sessions remained with a negative sign in the implementation of the SMS strategy (figure 2). According to the diary of the coordinator children and especially Fotis at the $1^{\text {st }}$ and $2^{\text {nd }}$ session was started to use the first component and wait for his partner opinion but, although he was agreed about his role (e.g. finder role) then he used to change his mind, change roles holding the patterns or trying to work on the roles himself. Both pairs shared practices only at $4^{\text {th }}$ and $5^{\text {th }}$ sessions. But they preferred to be competitors than cooperating with the other team. But the problem was that both children with ASD were able to 
solve problems in assembling but they were disrupted the cooperation climate while they had to share with the other pair.

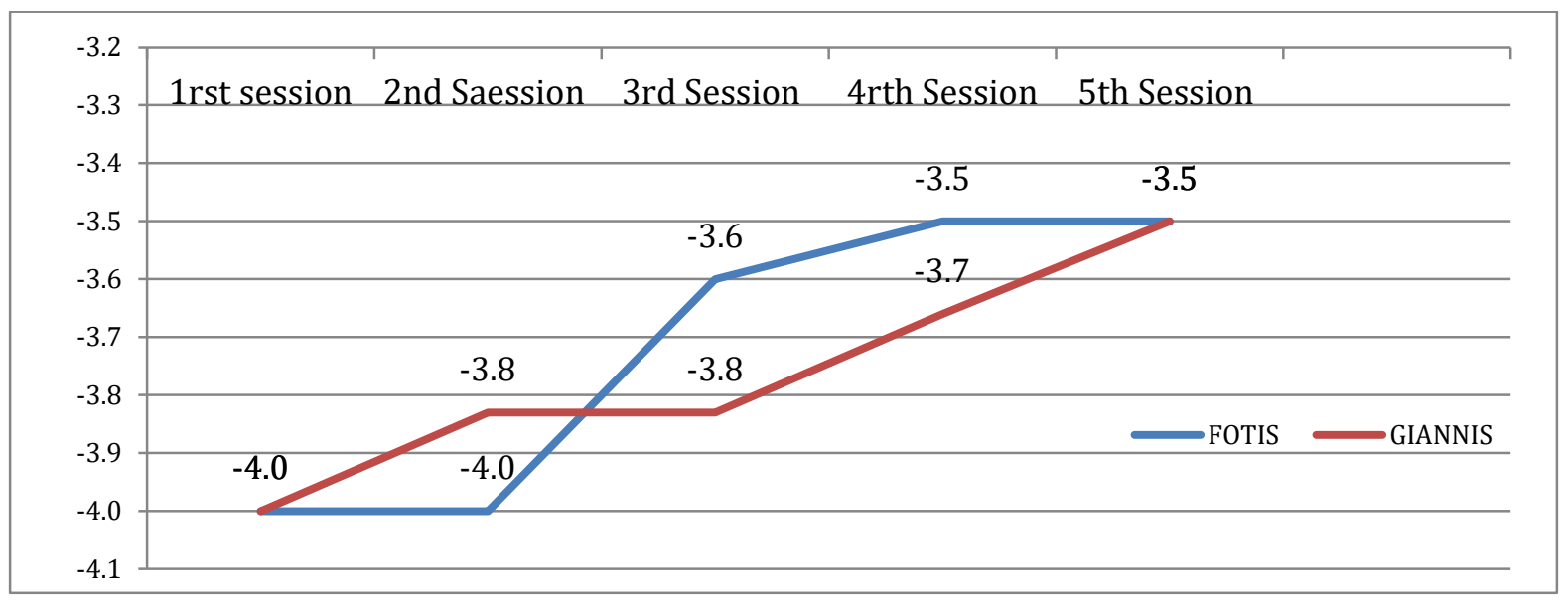

Figure 2 SMS Deviation of ASD children during the first 5 sessions

Reflect Their disrupted behaviors made the researchers worry. Additionally the researchers discussed ways to change attitudes of typical children to be more positive. How researchers would help ASD children to So the plan a new circle of Active research with extended goals.

$2^{\text {nd }}$ circle Plan: In the next sessions, children were taught to use in combination with SMS strategy more specific self- instruction and self assessment strategies. It was planned to be taught to ask themselves «Does my behavior help my team to assemble?” After every step they had to check if they have helped their team and hear of the opinion of their pair. Observe: According to the participant-researcher's diaries $\mathrm{F}$ was teasing the others or hitting his hand on the table. Then he was motivated to apply self regulated strategies and ask himself about his behavior. Children were motivated through coding processes and especially through the touch and ultrasonic sensors coding. During the 12 sessions assembling and coding aims have been achieved. As found be SDAP, the two children with ASD Fotis and Giannis in the two groups improved their cooperation skills and deviation from the strategy use significantly reduced $(-1,3$ and $-1,83)$. Reflect: Specific Self-regulation strategies and SMS strategy helped children with ASD to be more effective in cooperation. According to the coordinator researcher's diaries children were really motivated though the coding activities of EV3 robot and they were really find helpful to concentrate on SMS components in order to be positive and more engaged in these activities. Otherwise the process was disrupted and they were feeling disappointed. 
SOCIETY. INTEGRATION. EDUCATION

Proceedings of the International Scientific Conference. Volume IV, May $22^{\text {th }}-23^{\text {th }}, 2020.148-156$

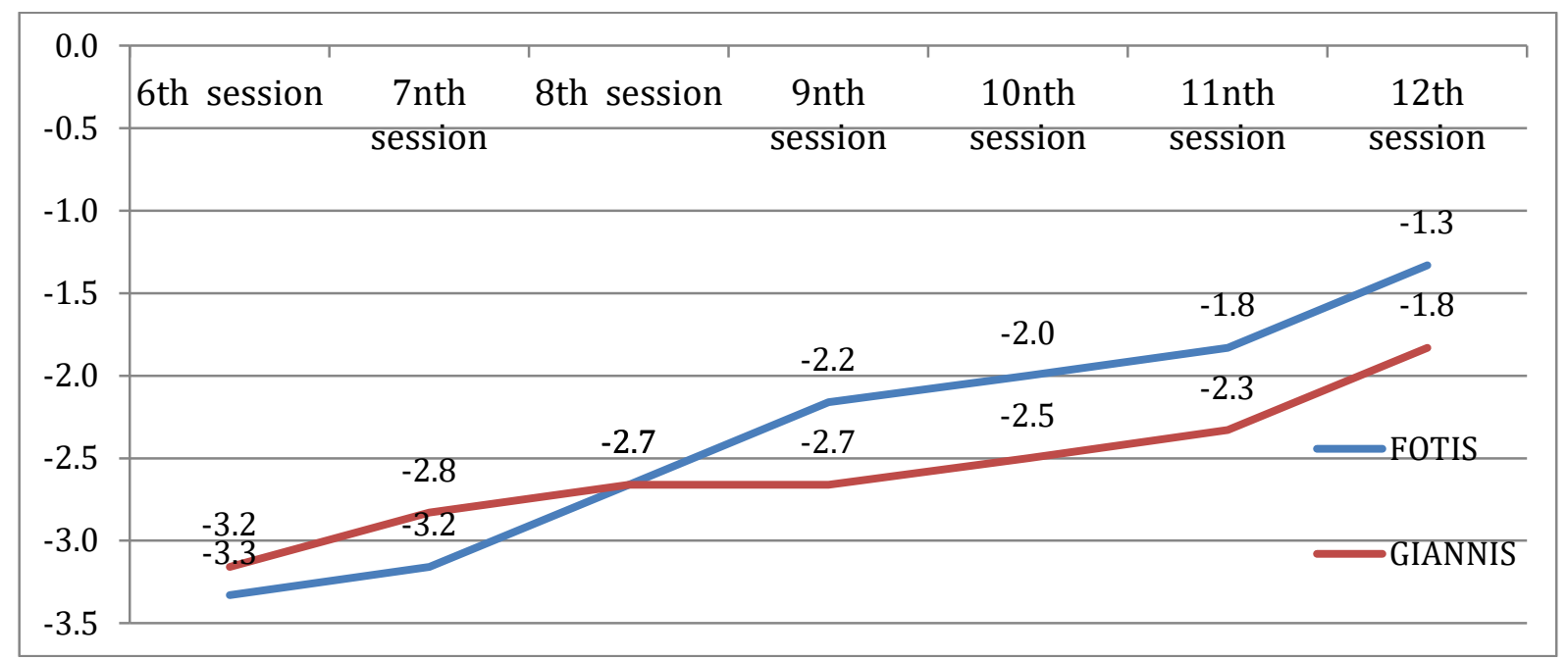

Figure 3 SDAP average of SMS strategy deviation from $6^{\text {th }}$ to $12^{\text {th }}$ session

During all sessions from $1^{\text {st }}$ to $12^{\text {th }}$ the deviation from strategy use was decreased. At the first session deviation from SMS strategy was -4 for both children at the $5^{\text {th }}$ became $-3,5$ and at the $12^{\text {th }}-1,3$ (Fotis) and $-1,8$ (Giannis) (Figure 4).

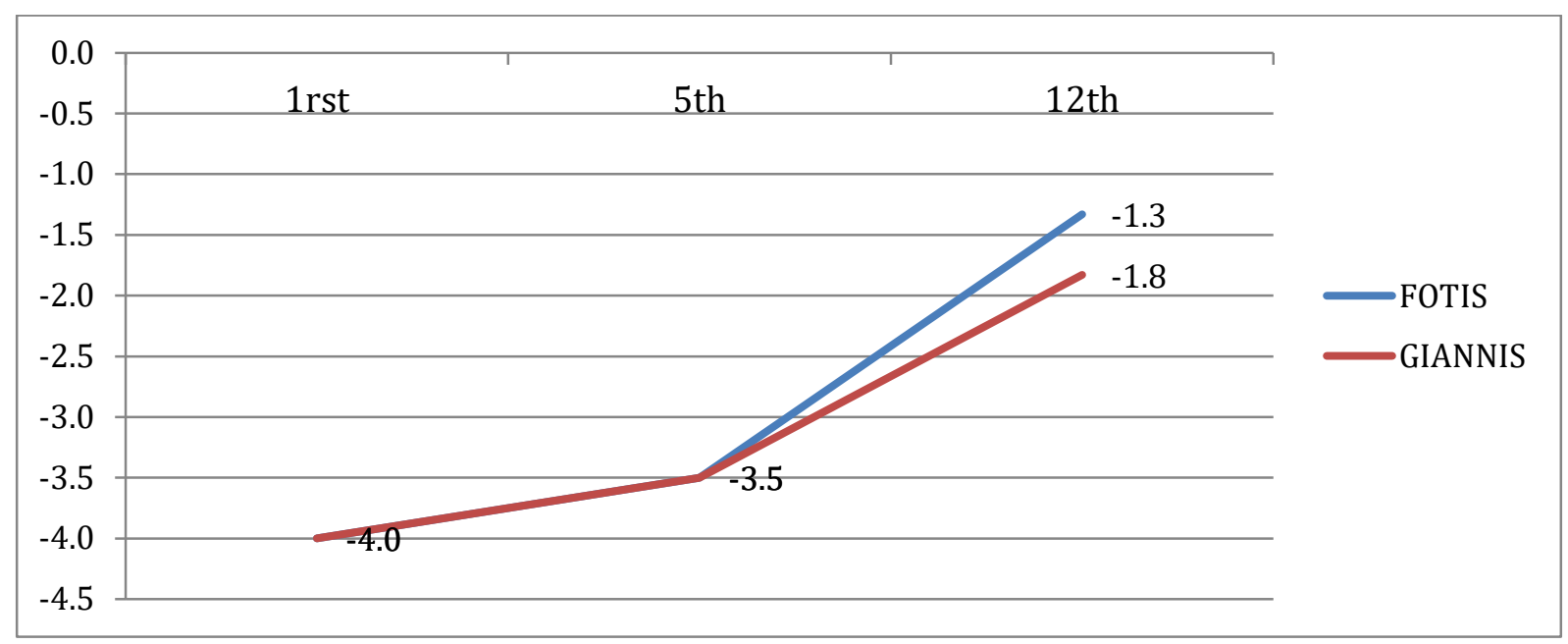

Figure 4 SDAP average of SMS strategy deviation at $1^{\text {st }}, 5^{\text {th }}$ and $12^{\text {th }}$ sessions

\section{Conclusions}

PTS cooperative strategy was not helpful for children to decide and take specific roles in assembling without self regulated and self assessment strategies. The SMS strategy and specific self regulated strategies that have been implemented using social story teaching approach although proved helpful in creating a climate of co-operation between typical and ASD children had 
difficulties in agreement about their roles in assembling. As previous research indicated students' interaction during brick assembling were mostly conversations on trivial tasks such as seeking bricks (Ching-Ching, Pei-Li, \& Kuo-Hung, 2013). Specific roles addressed by coordinator during assembling were effective in inclusive group (Nanou et al., 2019). Extending work on children's with ASD Cooperative Learning strategies this study found that specific strategies improved social behavior and cooperation with typical peers (Grey et al., 2007). Disrupted behaviors of ASD children bothered typical peers. Cooperative learning intervention in inclusive settings are of great importance for the development of social skills of children faces difficulties in cooperation.

Limitations and Future Research. Future research could investigate more effective self regulative strategies to foster cooperative ones in inclusive robotic activities. Empirical research on this field would lead to more effective inclusive practices in robotic activities. Typical children reactions had to be investigated in future research.

\section{References}

American Psychiatric Association. (2013). Diagnostic and statistical manual of mental disorders, Fifth Edition (DSM-5). Washington, DC: Author.

Ching-Ching, C., Pei-Li, H., \& Kuo-Hung, H. (2013). Cooperative Learning in Lego Robotics Projects: Exploring the Impacts of Group Formation on Interaction and Achievement. Journal of networks, 8(7), 1529-1535.

Drosinou, M. (2009). Research and Religious Affairs by Ministry of Education. Retrieved from http://www.pischools.gr/download/lessons/drast_math_etoimotitas/vivlio_ekp/vivlio.pd $\mathrm{f}$

Grey, I.M., Bruton, C., Honan, R., Mc Guinness, R., \& Daly, M. (2007). Co-operative Learning for Children with an Autistic Spectrum Disorder (ASD) in Mainstream and Special Class Settings: An exploratory study. Educational Psychology in Practice, 23(4), 317-327.

Gray, C.L. (1998). Social Stories and Comic Strip Conversations with Students with Asperger Syndrome and High-Functioning Autism. In E. Schopler, G.B. Mesibov \& L.J. Kunce (Eds.), Asperger Syndrome or High-Functioning Autism? (pp. 167-198). Boston: Springer.

Hinchliffe, K., Saggers, B., Chalmers, C., \& Hobbs, J. (2016). Utilising robotics social clubs to support the needs of students on the autism spectrum within inclusive school settings. Autism CRC, Brisbane: QLD.

Leman, P. (2015). How Do Groups Work? Age Differences in Performance and the Social Outcomes of Peer Collaboration. Cognitive Science, 39, 804-820.

Lyman, Frank. (1987). Think-Pair-Share: An Expanding Teaching Technique. MAA-CIE Cooperative News 1.1, 1-2.

Magos, K., \& Panagopoulou, P. (2008). I act by researching and I research by acting: an action research in teacher training. In the Proceedings of the $3^{\text {rd }}$ International Conference of Scientific Association of Adult Education: training and professional training of adult educators (CD - ROM). 
McConnell, S.R. (2002). Interventions to facilitate social interaction for young children with autism: Review of available research and recommendations for educational intervention and future research. Journal of Autism and Developmental Disorders, 32(5), 351-372.

McNiff, J. (1999). Action Research: Principles and Practices, London: Routledge.

Nanou, A., Chenine, I., \& Oikonomou, A. (2019). Using Lego-type robots to teach social skills in non -formal inclusive environment: an action research study. $24^{\text {th }}$ International Scientific Conference Educational Research and School Practice, Serbia.

Owens, G., Gordon, K., \& Baron-Cohen, S. (2008). Treating autism spectrum conditions. In J. Trafton \& W. Gordon (Eds.), Best practices in the behavioral management of health from precon- ception to adolescence (Vol. 3). Los Altos, CA: Institute for Brain Potential.

Reid, R., Mason, L., \& Asaro-Saddler, K. (2013). Self-Regulation Strategies for Students with Autism Spectrum Disorder. In S. Goldstei \& J.A Naglieri (Eds.), Interventions for Autism Spectrum Disorders (pp. 257-281). New York: Springer.

Reynolds, S., Bendixen, R., Lawrence, T., \& Lane, S. (2011). A Pilot Study Examining Activity Participation, Sensory Responsiveness, and Competence in Children with High Functioning Autism Spectrum Disorder. Journal Autism Dev Disorder, 41, 1496-1506.

Rogers, S.J. (2000). Interventions that facilitate socialization in children with autism. Journal of Autism and Developmental Disorders, 30(5), 399-409.

Volkmar, F.R., Paul, R., \& Rogers, S.J. (2014). Handbook of autism and pervasive developmental disorders ( $4^{\text {th }}$ ed.). NJ: John Wiley \& Sons.

Wang, J., \& Zhong, B. (2018). A preliminary study on switching roles in pair learning of robotics education. Proceedings of the 11th International Conference on Ubi-Media Computing and Workshops, 191-194.

Wehmeyer, M., Hughes, C., Agran, M., Garner, N., \& Yeager, D. (2003). Student-directed learning strategies to promote the progress of students with intellectual disability in inclusive classrooms. Inclusive Education, 7(4), 415-428. 